\title{
A Comprehensive Overview of the Paradigm of Laboratory Findings in the Diagnosis of the Pandemic Coronavirus Disease 2019 (COVID-19) in India
}

\author{
Ravi Gautam¹, Anshu Gupta ${ }^{2 *}$ \\ ${ }^{1}$ Department of Pathology, Swami Dayanand Hospital, Delhi, India \\ ${ }^{2}$ Department of Pathology and In-Charge Emergency Laboratory, Institute of Human Behavior and Allied Sciences (IHBAS), \\ Delhi, India \\ Email: *dransh2002@yahoo.co.in
}

How to cite this paper: Gautam, R. and Gupta, A. (2022) A Comprehensive Overview of the Paradigm of Laboratory Findings in the Diagnosis of the Pandemic Coronavirus Disease 2019 (COVID-19) in India. Open Journal of Pathology, 12, 12-21. https://doi.org/10.4236/ojpathology.2022.1 $\underline{21002}$

Received: March 24, 2021

Accepted: November 27, 2021

Published: November 30, 2021

Copyright $\odot 2022$ by author(s) and Scientific Research Publishing Inc. This work is licensed under the Creative Commons Attribution International License (CC BY 4.0).

http://creativecommons.org/licenses/by/4.0/

\begin{abstract}
COVID-19 is a pandemic disease caused by 2019 Novel Coronavirus or SARS-CoV-2. As this disease is highly contagious and rapidly spreading, so all countries have to increase their level of preparedness, alert, and response to identify, manage, and care for new cases of COVID-19; laboratory testing is an integral part of this strategy. Accurate results by laboratory diagnosis are beneficial for public health.
\end{abstract}

\section{Keywords}

COVID-19, SARS-CoV-2, Laboratory Diagnosis, Molecular Testing, Diagnosis, Diagnostic Tests

\section{Introduction}

\subsection{History and Background}

International Committee on Taxonomy of Viruses (ICTV) announced "severe acute respiratory syndrome coronavirus 2 (SARS-CoV-2)" as the name of the new virus on 11 February 2020. This name was chosen because the virus is genetically related to the coronavirus responsible for the SARS outbreak of 2003. WHO announced "COVID-19" as the name of this new disease on 11 February 2020 [1].

COVID 19 is an infectious disease caused by 2019 novel coronavirus (SARS-CoV-2). It is observed that some of the cases had a link to a wholesale food market in Wuhan and some did not. SARS-CoV-2 was identified in early 
January, 2020 and its genetic sequence shared publicly on 11-12 January, 2020. The full genetic sequence showed that SARS-CoV-2 has an ecological origin in bat populations. So, it is suggested that SARS-CoV-2 has a zoonotic source [2]. Since there is usually limited close contact between humans and bats, it is more likely that transmission of the virus to humans happened through another animal species which could be a domestic animal, a wild animal, or a domesticated wild animal and, as of yet, has not been identified [2].

\subsection{Incubation Period and Transmission}

Incubation period ranges from 2 to 14 days. It is transmitted by inhalation or contact with infected droplets [3]. SARS-CoV-2 nucleic acid can be detected in the feces and urine of patients with COVID-19 according to a study. It suggests that SARS-CoV-2 may be transmitted through the fecal-oral route [4].

\subsection{Clinical Features}

Manifestation of COVID-19 ranges from asymptomatic patients to septic shock and multiorgan dysfunction. Common symptoms include fever, fatigue, dry cough and diarrhea. On the basis of severity of presentation, COVID-19 is classified into mild, moderate, severe and critical. $81 \%$ of COVID-19 cases represent mild disease which includes upper respiratory tract viral infections like dry cough, mild fever, congestion, sore throat, headache, muscle pain and malaise. Moderate disease includes respiratory symptoms like cough, shortness of breath and tachypnea. Severe disease includes pneumonia, ARDS (acute respiratory disease syndrome), sepsis or septic shock with clinical manifestations of severe dyspnea, tachypnea (respiratory rate $>30 / \mathrm{min}$ ), respiratory distress with or without fever. 5\% of patients can develop critical disease with features of respiratory failure, RNAaemia, cardiac injury, septic shock, or multiple organ dysfunction [5].

\subsection{Pathogenesis}

SARS-CoV-2 virus is an enveloped positive single-stranded RNA (ssRNA) coronavirus. In the first open reading frame (ORF $1 \mathrm{a} / \mathrm{b}$ ), two-thirds of viral RNA is mainly located which encodes 16 non-structure proteins (NSPs) while the rest part of the virus genome encodes four essential structural proteins, including spike (S) glycoprotein, small envelope (E) protein, matrix $(\mathrm{M})$ protein, and nucleocapsid $(\mathrm{N})$ protein, and also several accessory proteins. Virus enters the host cell when $S$ glycoprotein binds with angiotensin-converting enzyme 2 (ACE2) ${ }^{5}$. Once the virus enters the cells, the viral RNA genome is released into the cytoplasm, it replicates and then cytokine storm occurs which causes the release of large amounts of pro-inflammatory cytokines [6].

\subsection{Comparison of SARS-CoV-2 Genome in India from That of Other Countries}

In one of the study, SARS-CoV-2 genome of India was compared with that of 
few other countries, and it was seen that Indian SARS-CoV-2 have mutations in orf1ab, nsp2, nsp3, helicase, ORF8 protein and spike surface glycoprotein. I296V, P1261L and T214I mutations in NSP2, NSP3 and helicase are specific to India SARS-CoV-2. It was also observed that out of all the miRNAs, hsa-miR-27b is the only unique miRNA specific to India SARS-CoV-2 and showed no significant complementarity-based nucleotide-binding with the strain of SARS-CoV-2 from other countries. This is surprising and is of utmost importance along with our other novel finding of a unique mutation identified in the spike surface glycoprotein [7].

\section{Methods}

A review of the literature was carried out in PubMed, searching for the articles with the terms "laboratory markers", "laboratory diagnosis", "haematological findings", "pathological findings", "novel coronavirus", "COVID-19 and SARS-CoV-2". Google Scholar searches have also been done by using the similar terms.

\section{Result}

The tests used in the diagnosis of COVID-19 include laboratory parameters, radiological findings and molecular findings.

\subsection{Laboratory Parameters}

Laboratory plays an important role in confirming the diagnosis, monitoring patient and epidemiological surveillance. Laboratory predictors help to assess disease severity and discriminating between severe and non severe cases.

\section{Hematology findings:}

Hematological investigations in most of the patients with COVID-19 show leucocytosis, absolute neutrophilia, lymphocytopenia, absolute monocytosis [8], thrombocytopenia, increased (NLR) Neutrophil/lymphocyte ratio (>3.13) [9], peak platelet/lymphocyte ratio and low lymphocyte/monocyte ratio. NLR is simple and less expensive biomarker to detect the progression of COVID-19. Leucocytosis is a sign of inflammatory response as a result of bacterial (super) infection. Neutrophilia occurs as a result of bacterial infection. Lymphocytes play an important role in inflammatory response and maintaining immune homoeostasis. Low lymphocyte \% is a predictor of prognosis in COVID-19 patients as it keeps on falling with severity of disease [10]. Lymphocytopenia indicates decreased immunological response to virus. Decrease in level of platelets reflects consumption or disseminated coagulopathy, seen in viral infection. An increase in level of coagulation profile including PT, aPTT, fibrinogen, and D-dimer shows activation of blood coagulations or disseminated coagulopathy and is predictors of disease severity [11].

Biochemistry findings:

There is an increased LDH (lactate dehydrogenase), raised AST, and ALT le- 
vels, increased total bilirubin, raised creatinine kinase and urea, raised inflammatory markers including increased ESR, C-reactive protein (CRP) and ferritin, increased cardiac troponins, and also increased procalcitonin (Table 1) [5]. LDH is increased in pulmonary injury. Increase level of ALT, AST and total bilirubin is an indicator of liver injury or organ failure. Raised creatinine and urea level are indicators of kidney failure. An increase in level of inflammatory markers including increased ESR, CRP is indicator of severe inflammation or tissue injury, severe viral infection, viremia, sepsis. Raised ferritin shows severe inflammation. Cardiac troponin is increased in cardiac injury. High procalcitonin indicates bacterial superinfection [11].

\subsection{Laboratory Findings Including Immunological Markers in Various Stages of the Disease}

Early stage:

In the early stages of the disease, there is high CRP, fibrinogen, and low lymphocyte and eosinophil found. Eosinophil is decreased due to restriction of its release in marrow, in response to the stress mechanism in case of acute lung injury in COVID-19 (reference-Early stage test). SARS-CoV-2 infection affects primarily $\mathrm{T}$ lymphocytes particularly $\mathrm{CD} 4+, \mathrm{CD} 8+\mathrm{T}$ cells which results in marked reduction in CD4 and CD8 lymphocytes.

Late stage:

Patients in the intensive care unit show progression of inflammatory response like higher levels of interleukin (IL) 2, IL-7, IL-10, GCSF (granulocyte colony-

Table 1. Laboratory findings in patients with COVID-19.

\begin{tabular}{cccc}
\hline \multicolumn{4}{c}{ Laboratory findings in COVID-19 Patients } \\
\hline Hematological changes & Biochemistry changes \\
\hline Leucocytes & Increased & LDH & Increased \\
Neutrophils & Increased & D-dimer & Increased \\
Lymphocytes & Decreased & ALT & Increased \\
Absolute Monocytes & Increased & \$AST & Increased \\
Platelets & Decreased & Creatinine kinase & Increased \\
ESR & Increased & Urea & Increased \\
Neutrophil/lymphocyte ratio & Increased & C-reactive protein & Increased \\
Platelet/lymphocyte ratio & Increased & Ferritin & Increased \\
& & Cardiac troponins & Increased \\
& & Procalcitonin & Increased \\
& & PT, aPTT, fibrinogen & Increased \\
& & Cytokines (IL6) & Increased \\
\hline
\end{tabular}

ESR-Erythrocyte sedimentation rate, LDH-Lactate dehydrogenase, ALT-Alanine aminotransferase, AST-Aspartate aminotransferase, PT-Prothrombin time, aPTTActivated partial thromboplastin time, IL6-Interleukin 6. 
stimulating factor), IP10 (interferon gamma-induced protein 10), MCP1 (monocyte chemotactic protein 1), MIP1A (macrophage inflammatory protein alpha), and TNF- $\alpha$ (tumor necrosis factor- $\alpha$ ). They also displayed other abnormal findings indicative of coagulation activation, cellular immune deficiency, myocardial injury, renal injury, and hepatic injury. In critical patients, amylase and D-dimer levels are significantly elevated. However, blood lymphocyte counts progressively decreased. Common to non-survivors are the elevations in ferritin, neutrophil count, D-dimer, cardiac troponin I, LDH, blood urea, blood urea nitrogen (BUN) and creatinine levels (Table 2) [5].

Table 2. Laboratory findings in early and late stages of COVID-19.

\begin{tabular}{|c|c|c|c|c|}
\hline \multirow[t]{2}{*}{ Laboratory Parameters } & \multirow{2}{*}{$\begin{array}{c}\text { Early stage of } \\
\text { COVID-19 }\end{array}$} & \multicolumn{3}{|c|}{ Late Stages of COVID-19 } \\
\hline & & ICU Patients & Critical & Non-survivors \\
\hline CD4 lymphocytes & Decreased & & & \\
\hline CD8 lymphocytes & Decreased & & & \\
\hline Interleukin IL-2, IL-7, IL-10 & & Increased & & \\
\hline GCSF & & Increased & & \\
\hline IP10 & & Increased & & \\
\hline MCP1 & & Increased & & \\
\hline MIP1A & & Increased & & \\
\hline TNF- $\alpha$ & & Increased & & \\
\hline $\mathrm{LDH}$ & & Increased & & \\
\hline D-dimer & & & Increased & Increased \\
\hline ALT & & Increased & & \\
\hline AST & & Increased & & \\
\hline Creatinine kinase & & Increased & & Increased \\
\hline Urea & & Increased & & Increased \\
\hline C-reactive protein & Increased & Increased & & \\
\hline Cardiac troponins & & Increased & & \\
\hline PT, aPTT & & Increased & Increased & \\
\hline Fibrinogen & Increased & Increased & & \\
\hline Eosinophil & Decreased & & & \\
\hline Amylase & & & Increased & \\
\hline Lymphocyte & Decreased & & Decreased & \\
\hline Ferritin & & & & Increased \\
\hline Neutrophil & & & & Increased \\
\hline BUN & & & & Increased \\
\hline
\end{tabular}

GCSF-Granulocyte colony-stimulating factor, IP10-Interferon gamma-induced protein 10 MCP1-Monocyte chemoattractant protein-1, MIP1A-Macrophage Inflammatory Protein 1 alpha, TNF $\alpha$-Tumor Necrosis Factor alpha, LDH-Lactate dehydrogenase, ALT-Alanine aminotransferase, AST-Aspartate aminotransferase, PT-Prothrombin time, aPTT-Activated partial thromboplastin time, BUN-Blood urea nitrogen. 


\subsection{Other Diagnostic Findings in COVID-19 Infection}

\section{Radiological findings}

Chest imaging modalities like chest X-ray, computed tomography (CT) scan, and lung ultrasound can be used to support the diagnosis of COVID-19. The most frequent finding on CT scan includes ground-glass opacity (86\%), consolidation (29\%), crazy paving (19\%), bilateral disease distribution (76\%), and peripheral disease distribution (33\%). It can also detect mediastinal lymphadenopathy, nodules, cystic changes, and pleural effusion [5].

\section{Molecular findings}

1) Nucleic acid detection technology: There are three methods to detect nucleic acid which are given below

a) Real-time quantitative polymerase chain reaction ( $R T-q P C R)$ and highthroughput sequencing

At the Indian Council of Medical Research-National Institute of Virology (ICMR-NIV), Pune, real-time RT-PCR assay has been used for screening ( $E$ gene assay) and confirmation ( $R d R p, N$ and $O R F$ gene) [12]. For high-throughput sequencing, two machines are installed at National Institute of Biologicals (NIB), Noida, Uttar Pradesh and Regional Medical Research Center (RMRC), Bhubaneshwar, Odisha [13].

b) Nucleic acid amplification tests (NAAT)-rRT-PCR

Routine confirmation of cases of COVID-19 is based on detection of unique sequences of virus RNA by NAAT such as real-time reverse-transcription polymerase chain reaction (rRT-PCR) with confirmation by nucleic acid sequencing which includes the N, E, S and RdRP genes [14].

c) RT-PCR test for the qualitative detection of nucleic acid from the SARS-CoV-2

The XPERT XPRESS SARS-COV-2 test is a rapid, real-time RT-PCR test intended for the qualitative detection of nucleic acid from the SARS-CoV-2 including N2 and E genes [15]. In India, real-time Polymerase Chain Reaction (RT-PCR) systems such as GeneXpert and Roche Cobas-6800/800, are to be used in emergency situations [16].

\section{2) Rapid antibody test:}

ICMR has suggested rapid antibody test for COVID-19 as a supplementary tool to assess the prevalence of the diseases within a specific area/perimeter but cannot replace the frontline test. It is useful after a minimum of 7 days of onset of symptoms. It is beneficial for epidemiological studies and surveillance purpose [16]. Biomedomics has developed rapid IgM-IgG combined antibody test for covid-19. It detects both early and late markers [17]. Chennai-based private sector enterprise, CPC Diagnostics Pvt. Ltd., has developed the Anti-SARS-CoV-2 ELISAs for IgG and IgA. The diagnostic test has higher sensitivity (up to 100\%) and specificity (up to 97.5\%) [18].

3) Rapid diagnostic tests based on antigen detection:

It detects the presence of viral proteins (antigens) expressed by the COVID-19 
virus in a sample from the respiratory tract of a person. If the target antigen is present in sufficient concentrations in the sample, it will bind to specific antibodies fixed to a paper strip enclosed in a plastic casing and generate a visually detectable signal, typically within 30 minutes. The antigen(s) detected are expressed only when the virus is actively replicating. Therefore, such tests are best used to identify acute or early infection. The sensitivity of these tests might be expected to vary from $34 \%$ to $80 \%$ based on experience with antigen-based RDTs for other respiratory diseases such as influenza.

WHO does not recommend the use of antigen-detecting rapid diagnostic tests and antibody-detecting rapid diagnostic tests for patient care but encourages the continuation of work to establish their usefulness in disease surveillance and epidemiologic research [19].

\section{Discussion}

The outbreak of coronavirus disease 2019 (COVID-19) has created a global health crisis. However, molecular identification is the gold standard for Coronavirus Disease 2019 (COVID-19) diagnosis, but common laboratory parameters also play a pivotal role in case detection [20]. COVID-19 cases are exponentially increasing posing a major challenge for treatment in ICU. Severe and critical cases of COVID-19 with acute respiratory distress (ARDS) and multi-organ failure require highly intensive care. So, timely detection of disease progression is crucial for appropriate management and intervention. There are several laboratory parameters which may facilitate the assessment of disease severity. These include low lymphocyte count as well as the serum levels of CRP, D-dimers, ferritin, cardiac troponin and IL-6 [21]. Based on analysis from several reports, lymphopenia is considered as a strong indicator of COVID-19 infection [20]. Lymphopenia is associated with disease severity as it is observed that lymphocyte counts are lower in non-survivor CVOID-19 patients than survivors [21]. Platelet count is another important hematological parameter in both diagnosis and prognosis. It is seen that in one of the study, thrombocyte count was significantly lower in COVID-19 compared to non-COVID-19 [20]. It is found that white blood cells, neutrophils, eosinophils, platelets, and CD8 cell counts are partial predictors in differentiating mild from severe COVID-19. Granulocyte colony stimulating factor (G-CSF) is related with disease severity as found to be elevated in ICU patients [20].

Signs of liver dysfunction including increase in alanine aminotransferase (ALT), aspartate aminotransferase (AST) and total bilirubin levels have been observed among many ICU patients suffering from COVID-19. C-reactive protein (CRP) levels are related with disease severity and prognosis as it is found to be increased in COVID-19 patients among nonsurvivors with median value of $125 \mathrm{mg} / \mathrm{L}$ as compared to survivor with median value of $40 \mathrm{mg} / \mathrm{L}$. Elevated ferritin levels have been reported in severe COVID-19 patients due to secondary hemophagocytic lymphohistiocytosis (sHLH) and cytokine storm syndrome. 
Higher levels of plasma D-dimers and fibrin degradation products, increased prothrombin times and activated partial thromboplastin are observed in non-survivors compared to survivors. Elevated cardiac troponin I levels indicate heart injury and are found be to because of mortality in critically ill patients [21].

In older patients and those with pre-existing illness like cardiovascular diseases, diabetes, respiratory diseases, it can progress to severe disease with critical respiratory symptoms. Higher concentrations of proinflammatory cytokines and increased secretion of T-helper-2 are found in COVID-19 patients admitted to ICUs. Tumour necrosis factor alpha (TNF- $\alpha$ ), interferon- $\gamma$-induced protein 10 (IP-10), monocyte chemoattractant protein 1 (MCP-1), chemokine (C-C motif), ligand 3 (CCL-3), and distinct interleukins (IL) -IL-2, IL-6, IL-7 and IL-10 are found to be associated with disease severity especially in cases admitted to ICUs. The haematological and biochemical findings stated here are globally observed phenomenon in COVID-19 patients [21]. There are some limitations in our study. First, because of the novelty of the outbreak, the quantity and diversity of the studies are limited. Further investigation is required to verify the findings in more diverse geographical settings, and resource conditions. Secondly a high heterogeneity was also noted in this study, which may be due to discrepancies between the classification between severe and non-severe COVID-19 across studies, and the lack of data regarding the exact timing of laboratory data collection in the studies. Hence future investigations need to assess the time-sensitive changes in laboratory parameters.

\section{Conclusion}

Hence, laboratory diagnosis plays an important role in detecting, thereby controlling the disease progression and transmission at an early stage. Molecular (e.g. PCR) testing of respiratory tract samples is the recommended method for the identification and laboratory confirmation of COVID-19 cases.

\section{Acknowledgements}

The author would like to thank Dr. Khushbu Gautam for assisting with related articles search and writing.

\section{Conflicts of Interest}

The authors declare no conflicts of interest.

\section{References}

[1] World Health Organization (2020) Global Name of COVID-19. https://www.who.int/emergencies/diseases/novel-coronavirus-2019/events-as-theyhappen

[2] World Health Organization (2020, April 23) Coronavirus Disease 2019 (COVID-19) Situation Report-94. https://www.who.int/emergencies/diseases/novel-coronavirus-2019/situation-reports

[3] Singhal, T. (2020) A Review of Coronavirus Disease-2019 (COVID-19). The Indian 
Journal of Pediatrics, 87, 281-286. https://doi.org/10.1007/s12098-020-03263-6

[4] Yang, P. and Wang, X. (2020) COVID-19: A New Challenge for Human Beings. Cellular \& Molecular Immunology, 17, 555-557.

https://doi.org/10.1038/s41423-020-0407-x

[5] Hassan, S., Sheikh, F.N., Jamal, S., Ezeh, J.K. and Akhtar, A. (2020) Coronavirus (COVID-19): A Review of Clinical Features, Diagnosis, and Treatment. Cureus, 12, Article ID: e7355. https://doi.org/10.7759/cureus.7355

[6] Li, X., Geng, M., Peng, Y., Meng, L. and Lu, S. (2020) Molecular Immune Pathogenesis and Diagnosis of COVID-19. Journal of Pharmaceutical Analysis, 10, 102-108. https://doi.org/10.1016/j.jpha.2020.03.001

[7] Sardar, R., Satish, D., Birla, S. and Gupta, D. (2020) Comparative Analyses of SAR-CoV2 Genomes from Different Geographical Locations and Other Coronavirus Family Genomes Reveals Unique Features Potentially Consequential to Host-Virus Interaction and Pathogenesis. Heliyon, 6, Article ID: E04658. https://doi.org/10.1016/j.heliyon.2020.e04658

[8] Kakodkar, P., Kaka, N. and Baig, M. (2020) A Comprehensive Literature Review on the Clinical Presentation, and Management of the Pandemic Coronavirus Disease 2019 (COVID-19). Cureus, 12, Article No. e7560. https://doi.org/10.7759/cureus.7560

[9] Liu, J., Liu, Y., Xiang, P., Xiong, H., Li, C., Zhang, M., et al. (2020) Neutrophil to Lymphocyte Ratio Predicts Severe Illness Patients with 2019 Novel Coronavirus in the Early Stage. Journal of Translational Medicine, 18, Article No. 206. https://doi.org/10.1186/s12967-020-02374-0

[10] Terpos, E., Ntanasis-Stathopoulos, I., Elalamy, I., Kastritis, E., Sergentanis, T.N., Politou, M., et al. (2020) Hematological Findings and Complications of COVID-19. American Journal of Hematology, 95, 834-847. https://doi.org/10.1002/ajh.25829

[11] IFCC (International Federation of Clinical Chemistry and Laboratory Medicine) (2020) IFCC Information Guide on COVID-19. https://www.ifcc.org/ifcc-news/2020-03-26-ifcc-information-guide-on-covid-19/

[12] Choudhary, M.L., Vipat, V., Jadhav, S., Basu, A., Cherian, S., Abraham, P., et al. (2020) Development of In Vitro Transcribed RNA as Positive Control for Laboratory Diagnosis of SARS-CoV-2 in India. Indian Journal of Medical Research, 151, 251-254.

[13] Gupta, N., Bhatnagar, T., Rade, K., Murhekar, M., Gangakhedkar, R.R. and Nagar, A. (2020) Strategic Planning to Augment the Testing Capacity for COVID-19 in India. Indian Journal of Medical Research, 151, 210-215.

[14] World Health Organization (2020) Laboratory Testing for Coronavirus Disease (COVID-19) in Suspected Human Cases. World Health Organization, Geneva. https://apps.who.int/iris/handle/10665/331329

[15] Xpert $^{\oplus}$ Xpress SARS-CoV-2. Gene Xpert. Cepheid 2020 Apr; 1-18.

[16] ICMR (Indian Council of Medical Research) (2020) Protocol for Using "Rapid Antibody Test" in Hot Area-Epidemiological Studies and Surveillance. Indian Council of Medical Research, New Delhi. https://www.icmr.gov.in/pdf/covid/strategy/Rapid Antibody test Protocol.pdf

[17] BioMedomics (2020) COVID-19 IgM/IgG Rapid Test. https://www.biomedomics.com/products/infectious-disease/covid-19-rt/

[18] Euroimmun (2020) Anti-SARS-CoV-2 ELISAs for IgG and IgA. Euroimmun, Lubeck. https://cpcdiagnostics.in/sars-cov-2.php 
[19] World Health Organization (2020) Advice on the Use of Point-of-Care Immunodiagnostic Tests for COVID-19.

https://www.who.int/news-room/commentaries/detail/advice-on-the-use-of-pointof-care-immunodiagnostic-tests-for-covid-19

[20] Soraya, G.V. and Ulhaq, Z.S. (2020) Crucial Laboratory Parameters in Covid-19 Diagnosis and Prognosis: An Updated Meta-Analysis. SSRN Electronic Journal, 1-20. https://doi.org/10.2139/ssrn.3576912

[21] Velavana, T.P. and Meyera, C.G. (2020) Mild versus Severe COVID-19: Laboratory Markers. International Journal of Infectious Diseases, 95, 304-307. https://doi.org/10.1016/j.ijid.2020.04.061 